

\title{
Three-dimensional quadrics in extended conformal geometric algebras of higher dimensions from control points, implicit equations and axis alignment
}

Stéphane Breuils, Laurent Fuchs, Eckhard Hitzer, Vincent Nozick, Akihiro Sugimoto

\section{To cite this version:}

Stéphane Breuils, Laurent Fuchs, Eckhard Hitzer, Vincent Nozick, Akihiro Sugimoto. Threedimensional quadrics in extended conformal geometric algebras of higher dimensions from control points, implicit equations and axis alignment. Advances in Applied Clifford Algebras, 2019, 29 (3), 10.1007/s00006-019-0974-z . hal-02169419

\author{
HAL Id: hal-02169419 \\ https://hal.science/hal-02169419
}

Submitted on 27 Jul 2019

HAL is a multi-disciplinary open access archive for the deposit and dissemination of scientific research documents, whether they are published or not. The documents may come from teaching and research institutions in France or abroad, or from public or private research centers.
L'archive ouverte pluridisciplinaire HAL, est destinée au dépôt et à la diffusion de documents scientifiques de niveau recherche, publiés ou non, émanant des établissements d'enseignement et de recherche français ou étrangers, des laboratoires publics ou privés. 


\title{
Three-dimensional quadrics in extended con- formal geometric algebras of higher dimen- sions from control points, implicit equations and axis alignment
}

\author{
Stéphane Breuils, Laurent Fuchs, Eckhard Hitzer, Vincent \\ Nozick and Akihiro Sugimoto
}

\begin{abstract}
We introduce the quadric conformal geometric algebra (QCGA) inside the algebra of $\mathbb{R}^{9,6}$. In particular, this paper presents how three-dimensional quadratic surfaces can be defined by the outer product of conformal geometric algebra points in higher dimensions, or alternatively by a linear combination of basis vectors with coefficients straight from the implicit quadratic equation. These multivector expressions code all types of quadratic surfaces in arbitrary scale, location, and orientation. Furthermore, we investigate two types of definitions of axis aligned quadric surfaces, from contact points and dually from linear combinations of $\mathbb{R}^{9,6}$ basis vectors.
\end{abstract}

\section{Introduction}

Geometric algebra provides convenient and intuitive tools to represent, transform, and intersect geometric objects. Deeply explored by physicists, it has been used in quantum mechanics and electromagnetism [9, 8] as well as in classical mechanics [10]. Geometric algebra has also found interesting applications in geographic data manipulations $[16,20]$. Among them, geometric algebra is used within the computer graphics community. More precisely, it is used not only in basis geometric primitive manipulations [19] but also in complex illumination processes as in [17] where spherical harmonics are substituted by geometric algebra entities. Finally, in data and image analysis, we can find the usefulness of geometric algebra in mathematical morphology [4] and in neural networking [3, 12]. In the geometric algebra community, quadratic surfaces gain more and more attention and some frameworks have been proposed in order to represent, transform, and intersect these quadratic surfaces. 
There exist three main approaches to deal with quadratic surfaces. The first one, introduced in [6], is called double conformal geometric algebra of $\mathbb{G}_{8,2}$. It is capable of representing quadratic surfaces from the coefficients of their implicit form. The second one is double perspective geometric algebra of $\mathbb{G}_{4,4}$ whose definition was firstly introduced in [7]. It has been further developed in [5]. This approach is based on a duplication of $\mathbb{R}^{3}$ and also represents quadratic surfaces from the coefficients of their implicit form, as bivectors. The third one was introduced in [2] and is denoted as quadric conformal geometric algebra (QCGA). QCGA allows us to define general quadratic surfaces from nine control points, and to represent the objects in low dimensional subspaces of the algebra. With slight modifications, QCGA is also capable of constructing quadratic surfaces either using control points or implicit equations as 1-vector. QCGA also offers the possibility to transform quadratic surfaces using versors for rotation, translation and scaling [13].

In order to enhance usefulness of QCGA for geometry and computer graphics community, the QCGA framework must be further equipped with convenient tools and handy notations. This is the main purpose of this paper.

All the examples and computations are based upon the efficient geometric algebra library generator Garamon [1]. The code of this library generator is freely available online ${ }^{1}$.

The paper is organized as follows. Section 2 defines QCGA following [2], and the modifications introduced in [13]. It also includes a concise set of important algebraic relations in QCGA, handy for the computations in the rest of this work. Section 3 defines the fundamental notion of point in QCGA (identical to the one given in [2] and [13]), and reviews how the well-known range of geometric objects of conformal geometric algebra (CGA) can be successfully embedded, constructed and computed with in QCGA. Section 4 then introduces the general algebraic construction of quadratic surfaces from contact points. Next, the first main Section 5 concentrates on presenting the treatment of axis aligned quadratic surfaces defined from suitable contact points and null basis infinity vectors. The second main Section 6 treats the dual representation of quadratic surfaces in QCGA, which proves ideal for the straightforward definition of quadratic surfaces in terms of dual 1-vectors, simply constituting of linear combinations of basis vectors of $\mathbb{R}^{9,6}$ with coefficients from their implicit scalar equations. For completeness, Section 7 briefly reviews the way quadratic surfaces can be intersected in QCGA. Section 8 concludes the paper followed by acknowledgments and references.

\subsection{Contributions}

We provide new tools for QCGA that bring easier definition of conformal geometric algebra objects. With this construction, the definition of quadratic surfaces becomes more intuitive. We also show that QCGA is capable of defining both degenerate and non-degenerate centred axis-aligned quadratic surfaces from the minimum number of necessary control points. We also present an alternative way of definition, that makes direct use of the coefficients of the implicit quadratic equations.

\footnotetext{
${ }^{1}$ git clone https://git.renater.fr/garamon.git
} 


\subsection{Notation conventions}

Throughout this paper, the following notation is used: Lower-case bold letters denote basis blades and multivectors (multivector a). Italic lower-case letters refer to multivector components $\left(a_{1}, x, y^{2}, \cdots\right)$. For example, $a_{i}$ is the $i^{\text {th }}$ coordinate of the multivector a. Constant scalars are denoted using lower-case default text font (constant radius $r$ ) or simply $r$. The superscripts star used in $\mathbf{x}^{*}$ represents the dualization of the multivector $\mathbf{x}$. Moreover, subscript $\varepsilon$ on $\mathbf{x}_{\varepsilon}$ concerns the Euclidean vector associated with the vector $\mathbf{x}$ of QCGA. Finally, subscript $C$ refers to the embedding of Conformal Geometric Algebra of the entity.

Note that when used in the geometric algebra inner product, the contraction and the outer product have priority over the full geometric product. For instance, $\mathbf{a} \wedge \mathbf{b I}=(\mathbf{a} \wedge \mathbf{b}) \mathbf{I}$.

\section{QCGA definition and algebraic relations}

\subsection{QCGA basis and metric}

The algebraic equations in this section can be either computed by hand through expanding all blades in terms of basis vectors [11], or computed using a software such as the Clifford toolbox for MATLAB[18]. Further algebraic details and relationships may be found in Section 2 of [13]. The QCGA $C l(9,6)$ is defined over the 15 -dimensional vector space $\mathbb{R}^{9,6}$. The base vectors of the space are naturally divided into three groups: $\left\{\mathbf{e}_{1}, \mathbf{e}_{2}, \mathbf{e}_{3}\right\}$ (corresponding to Euclidean vectors of $\mathbb{R}^{3}$ ), $\left\{\mathbf{e}_{o 1}, \mathbf{e}_{o 2}, \mathbf{e}_{o 3}, \mathbf{e}_{o 4}, \mathbf{e}_{o 5}, \mathbf{e}_{o 6}\right\}$, and $\left\{\mathbf{e}_{\infty 1}, \mathbf{e}_{\infty 2}, \mathbf{e}_{\infty 3}, \mathbf{e}_{\infty 4}, \mathbf{e}_{\infty 5}, \mathbf{e}_{\infty 6}\right\}$. The inner products between them are defined in Table 1 .

TABLE 1. Inner product between QCGA basis vectors.

\begin{tabular}{|c|c|c|c|c|c|c|c|c|c|c|c|c|c|c|c|}
\hline & $a_{1}$ & $\mathbf{e}_{2}$ & $\mathbf{e}_{3}$ & $\mathbf{e}_{o 1}$ & $\mathbf{e}_{\infty 1}$ & $\mathbf{e}_{o 2}$ & $\mathbf{e}_{\infty 2}$ & $\mathbf{e}_{o 3}$ & $\mathbf{e}_{\infty 3}$ & $\mathbf{e}_{o 4}$ & $\mathbf{e}_{\infty 4}$ & $\mathbf{e}_{o 5}$ & $\mathbf{e}_{\infty 5}$ & $\mathbf{e}_{o 6}$ & $\mathbf{e}_{\infty 6}$ \\
\hline $\mathbf{e}_{1}$ & 1 & 0 & 0 & & . & . & $\cdot$ & . & 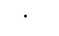 & . & . & 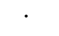 & . & $\cdot$ & \\
\hline $\mathbf{e}_{2}$ & 0 & 1 & 0 & & . & . & $\cdot$ & . & . & . &  & $\theta$ & . & $\cdot$ & \\
\hline $\mathbf{e}_{3}$ & 0 & 0 & 1 & & &  & $\cdot$ & . & . & 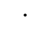 & 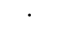 & 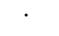 & . & $\cdot$ & \\
\hline $\mathbf{e}_{o 1}$ & 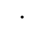 & $\cdot$ & 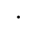 & 0 & -1 & 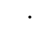 & $\cdot$ & . & . & . & · & . & . & $\cdot$ & \\
\hline $\mathbf{e}_{\infty 1}$ & . & $\cdot$ & $\cdot$ & -1 & 0 & . & $\cdot$ & . & . & . & . & . & . & $\cdot$ & \\
\hline $\mathbf{e}_{o 2}$ & . & $\cdot$ & $\cdot$ & 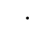 & . & 0 & -1 & . & . & . & . & . & . & $\cdot$ & \\
\hline $\mathbf{e}_{\infty 2}$ & . & $\cdot$ & $\cdot$ & & 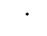 & -1 & 0 & . & . & . & . & . & . & $\cdot$ & \\
\hline $\mathbf{e}_{o 3}$ & · & $\cdot$ & $\cdot$ & & · & . & $\cdot$ & 0 & -1 & . & . & . & . & . & \\
\hline $\mathbf{e}_{\infty 3}$ & · & $\cdot$ & $\cdot$ & . & . & . & $\cdot$ & -1 & 0 & . & . & $\cdot$ & . & $\cdot$ & \\
\hline $\mathbf{e}_{o 4}$ & . & $\cdot$ & $\cdot$ & . & 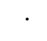 & . & $\cdot$ & . &  & 0 & -1 & . & . & . & . \\
\hline $\mathbf{e}_{\infty 4}$ & · & $\cdot$ & $\cdot$ & . & . & . & $\cdot$ & . & . & -1 & 0 & . & . & . & \\
\hline $\mathbf{e}_{o 5}$ & . & $\cdot$ & $\cdot$ & . & . & . & $\cdot$ & . & . & . & . & 0 & -1 & . & \\
\hline $\mathbf{e}_{\infty 5}$ & . & $\cdot$ & $\cdot$ & . & . & . & $\cdot$ & . & . & . & . & -1 & 0 & $\cdot$ & . \\
\hline $\mathbf{e}_{o 6}$ & 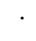 & $\cdot$ & 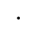 & . & · & . & $\cdot$ & 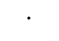 & 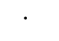 & . & . & . & . & 0 & -1 \\
\hline $\mathbf{e}_{\infty 6}$ & . & & . & . & . & . & . & . & . & . & . & . & . & -1 & 0 \\
\hline
\end{tabular}

For efficient computations, a diagonal metric matrix may furthermore be useful. The algebra $C l(9,6)$ generated by the Euclidean basis $\left\{\mathbf{e}_{1}, \mathbf{e}_{2}, \mathbf{e}_{3}\right\}$, and six basis vectors $\left\{\mathbf{e}_{+1}, \mathbf{e}_{+2}, \mathbf{e}_{+3}, \mathbf{e}_{+4}, \mathbf{e}_{+5}, \mathbf{e}_{+6}\right\}$ squaring to +1 along with six other basis vectors $\left\{\mathbf{e}_{-1}, \mathbf{e}_{-2}, \mathbf{e}_{-1}, \mathbf{e}_{-4}, \mathbf{e}_{-5}, \mathbf{e}_{-6}\right\}$ squaring to -1 , would correspond to a diagonal 
metric matrix. Following the approach of [13] for a successful formulation of versors for rotation, translation and scaling, the transformation from the diagonal metric basis to that of Table 1 can consistently be defined for $1 \leq i, j \leq 6$ as follows:

$$
\begin{aligned}
\mathbf{e}_{\infty i} & =\frac{1}{\sqrt{2}}\left(\mathbf{e}_{+i}+\mathbf{e}_{-i}\right), \quad \mathbf{e}_{o i}=\frac{1}{\sqrt{2}}\left(\mathbf{e}_{-i}-\mathbf{e}_{+i}\right), \quad \mathbf{e}_{\infty i} \cdot \mathbf{e}_{o i}=-1, \\
\mathbf{e}_{\infty} & =\frac{1}{3}\left(\mathbf{e}_{\infty 1}+\mathbf{e}_{\infty 2}+\mathbf{e}_{\infty 3}\right), \quad \mathbf{e}_{o}=\mathbf{e}_{o 1}+\mathbf{e}_{o 2}+\mathbf{e}_{o 3}, \\
\mathbf{e}_{\infty} \cdot \mathbf{e}_{o} & =-1, \quad \mathbf{e}_{o}^{2}=\mathbf{e}_{\infty}^{2}=0,
\end{aligned}
$$

with bivectors $E_{i}, E$, defined by

$$
\begin{gathered}
E_{i}=\mathbf{e}_{\infty i} \wedge \mathbf{e}_{o i}=\mathbf{e}_{+i} \mathbf{e}_{-i}, \quad E_{i}^{2}=1, \quad E_{i} E_{j}=E_{j} E_{i}, \\
\mathbf{e}_{o i} E_{i}=-E_{i} \mathbf{e}_{o i}=-\mathbf{e}_{o i}, \quad \mathbf{e}_{\infty i} E_{i}=-E_{i} \mathbf{e}_{\infty i}=\mathbf{e}_{\infty i}, \\
E=\mathbf{e}_{\infty} \wedge \mathbf{e}_{o}, \quad E^{2}=1, \quad \mathbf{e}_{o} E=-E \mathbf{e}_{o}=-\mathbf{e}_{o}, \quad \mathbf{e}_{\infty} E=-E \mathbf{e}_{\infty}=\mathbf{e}_{\infty} .
\end{gathered}
$$

For clarity, we also define the following blades:

$$
\begin{aligned}
\mathbf{I}_{\infty a} & =\mathbf{e}_{\infty 1} \mathbf{e}_{\infty 2} \mathbf{e}_{\infty 3}, \mathbf{I}_{\infty b}=\mathbf{e}_{\infty 4} \mathbf{e}_{\infty 5} \mathbf{e}_{\infty 6}, \mathbf{I}_{\infty}=\mathbf{I}_{\infty a} \mathbf{I}_{\infty b}, \\
\mathbf{I}_{o a} & =\mathbf{e}_{o 1} \mathbf{e}_{o 2} \mathbf{e}_{o 3}, \mathbf{I}_{o b}=\mathbf{e}_{o 4} \mathbf{e}_{o 5} \mathbf{e}_{o 6}, \mathbf{I}_{o}=\mathbf{I}_{o a} \mathbf{I}_{o b}, \\
\mathbf{I}_{\infty o} & =\mathbf{I}_{\infty} \wedge \mathbf{I}_{o}=-E_{1} E_{2} E_{3} E_{4} E_{5} E_{6}, \quad \mathbf{I}_{\infty o}^{2}=1, \\
\mathbf{I}_{o} \mathbf{I}_{\infty o} & =\mathbf{I}_{\infty o} \mathbf{I}_{o}=-\mathbf{I}_{o}, \quad \mathbf{I}_{\infty} \mathbf{I}_{\infty o}=\mathbf{I}_{\infty o} \mathbf{I}_{\infty}=-\mathbf{I}_{\infty}, \\
\mathbf{I}_{\infty a}^{\triangleright} & =\left(\mathbf{e}_{\infty 1}-\mathbf{e}_{\infty 2}\right) \wedge\left(\mathbf{e}_{\infty 2}-\mathbf{e}_{\infty 3}\right), \quad \mathbf{I}_{\infty}^{\triangleright}=\mathbf{I}_{\infty a}^{\triangleright} \mathbf{I}_{\infty b}, \\
\mathbf{I}_{o a}^{\triangleright} & =\left(\mathbf{e}_{o 1}-\mathbf{e}_{o 2}\right) \wedge\left(\mathbf{e}_{o 2}-\mathbf{e}_{o 3}\right), \quad \mathbf{I}_{o}^{\triangleright}=\mathbf{I}_{o a}^{\triangleright} \mathbf{I}_{o b}, \quad \mathbf{I}^{\triangleright}=\mathbf{I}_{\infty}^{\triangleright} \wedge \mathbf{I}_{o}^{\triangleright} .
\end{aligned}
$$

We note that

$$
\begin{aligned}
\mathbf{I}_{\infty a} \wedge \mathbf{I}_{o a} & =-E_{1} E_{2} E_{3}, \quad \mathbf{I}_{\infty b} \wedge \mathbf{I}_{o b}=-E_{4} E_{5} E_{6}, \\
\mathbf{I}^{\triangleright} & =\mathbf{I}_{\infty a}^{\triangleright} \wedge \mathbf{I}_{o a}^{\triangleright} \mathbf{I}_{\infty b} \wedge \mathbf{I}_{o b}=-\mathbf{I}_{\infty a}^{\triangleright} \wedge \mathbf{I}_{o a}^{\triangleright} E_{4} E_{5} E_{6}, \\
\left(\mathbf{I}^{\triangleright}\right)^{2} & =\left(\mathbf{I}_{\infty a}^{\triangleright} \wedge \mathbf{I}_{o a}^{\triangleright}\right)^{2}=9, \quad\left(\mathbf{I}^{\triangleright}\right)^{-1}=\frac{1}{9} \mathbf{I}^{\triangleright}, \\
\left(\mathbf{I}_{\infty a}^{\triangleright} \wedge \mathbf{I}_{o a}^{\triangleright}\right)^{-1} & =\frac{1}{9} \mathbf{I}_{\infty a}^{\triangleright} \wedge \mathbf{I}_{o a}^{\triangleright}, \\
\mathbf{I}_{\infty}^{\triangleright} \cdot \mathbf{I}_{o}^{\triangleright} & =\mathbf{I}_{o}^{\triangleright} \cdot \mathbf{I}_{\infty}^{\triangleright}=\mathbf{I}_{\infty}^{\triangleright} \mid \mathbf{I}_{o}^{\triangleright}=\mathbf{I}_{\infty}^{\triangleright}\left[\mathbf{I}_{o}^{\triangleright}=-3 .\right.
\end{aligned}
$$

We have the following outer products

$$
\begin{aligned}
\mathbf{I}_{\infty a} & =\mathbf{e}_{\infty 1} \wedge \mathbf{I}_{\infty a}^{\triangleright}=\mathbf{e}_{\infty 2} \wedge \mathbf{I}_{\infty a}^{\triangleright}=\mathbf{e}_{\infty 3} \wedge \mathbf{I}_{\infty a}^{\triangleright}=\mathbf{e}_{\infty} \wedge \mathbf{I}_{\infty a}^{\triangleright}=\mathbf{e}_{\infty} \mathbf{I}_{\infty a}^{\triangleright}, \\
\mathbf{I}_{o a} & =\mathbf{e}_{o 1} \wedge \mathbf{I}_{o a}^{\triangleright}=\mathbf{e}_{o 2} \wedge \mathbf{I}_{o a}^{\triangleright}=\mathbf{e}_{o 3} \wedge \mathbf{I}_{o a}^{\triangleright}=\frac{1}{3} \mathbf{e}_{o} \wedge \mathbf{I}_{o a}^{\triangleright}=\frac{1}{3} \mathbf{e}_{o} \mathbf{I}_{o a}^{\triangleright}, \\
\mathbf{I}_{\infty a} \wedge \mathbf{I}_{o a} & =E_{1} \wedge \mathbf{I}_{\infty a}^{\triangleright} \wedge \mathbf{I}_{o a}^{\triangleright}=E_{2} \wedge \mathbf{I}_{\infty a}^{\triangleright} \wedge \mathbf{I}_{o a}^{\triangleright}=E_{3} \wedge \mathbf{I}_{\infty a}^{\triangleright} \wedge \mathbf{I}_{o a}^{\triangleright} \\
& =\frac{1}{3} E \wedge \mathbf{I}_{\infty a}^{\triangleright} \wedge \mathbf{I}_{o a}^{\triangleright}=\frac{1}{3} E \mathbf{I}_{\infty a}^{\triangleright} \wedge \mathbf{I}_{o a}^{\triangleright} .
\end{aligned}
$$


And we have the following inner products

$$
\begin{aligned}
& \mathbf{I}_{o a}^{\triangleright}=-3 \mathbf{e}_{\infty} \cdot \mathbf{I}_{o a}, \quad \mathbf{I}_{o}^{\triangleright}=-3 \mathbf{e}_{\infty} \cdot \mathbf{I}_{o}, \\
& \mathbf{I}_{\infty a}^{\triangleright}=-\mathbf{e}_{o} \cdot \mathbf{I}_{\infty a}, \quad \mathbf{I}_{\infty}^{\triangleright}=-\mathbf{e}_{o} \cdot \mathbf{I}_{\infty}, \\
& \left(\mathbf{e}_{o i} \cdot \mathbf{I}_{\infty}\right) \cdot \mathbf{I}_{o}=-\mathbf{e}_{o i}, \quad\left(\mathbf{e}_{\infty i} \cdot \mathbf{I}_{o}\right) \cdot \mathbf{I}_{\infty}=-\mathbf{e}_{\infty i}, \\
& \left(\mathbf{e}_{o} \cdot \mathbf{I}_{\infty}\right) \cdot \mathbf{I}_{o}=-\mathbf{e}_{o}, \quad\left(\mathbf{e}_{\infty} \cdot \mathbf{I}_{o}\right) \cdot \mathbf{I}_{\infty}=-\mathbf{e}_{\infty} \\
& \mathbf{e}_{\infty} \cdot \mathbf{I}_{\infty O}=-\frac{1}{3} \mathbf{I}_{\infty} \wedge \mathbf{I}_{o}^{\triangleright}, \quad \mathbf{e}_{o} \cdot \mathbf{I}_{\infty o}=-\mathbf{I}_{\infty}^{\triangleright} \wedge \mathbf{I}_{o}, \\
& \mathbf{e}_{\infty i} \cdot \mathbf{I}_{\infty a}^{\triangleright}=0, \quad \mathbf{e}_{\infty i} \cdot \mathbf{I}_{\infty}^{\triangleright}=0, \quad \mathbf{e}_{\infty} \cdot \mathbf{I}_{\infty a}^{\triangleright}=0, \quad \mathbf{e}_{\infty} \cdot \mathbf{I}_{\infty}^{\triangleright}=0, \\
& \mathbf{e}_{o i} \cdot \mathbf{I}_{o a}^{\triangleright}=0, \quad \mathbf{e}_{o i} \cdot \mathbf{I}_{o}^{\triangleright}=0, \quad \mathbf{e}_{o} \cdot \mathbf{I}_{o a}^{\triangleright}=0, \quad \mathbf{e}_{o} \cdot \mathbf{I}_{o}^{\triangleright}=0, \\
& \mathbf{e}_{\infty} \cdot \mathbf{I}_{o a}^{\triangleright}=0, \quad \mathbf{e}_{\infty} \cdot \mathbf{I}_{o}^{\triangleright}=0, \quad \mathbf{e}_{o} \cdot \mathbf{I}_{\infty a}^{\triangleright}=0, \quad \mathbf{e}_{o} \cdot \mathbf{I}_{\infty}^{\triangleright}=0, \\
& \mathbf{e}_{\infty} \cdot \mathbf{I}^{\triangleright}=0, \quad \mathbf{e}_{o} \cdot \mathbf{I}^{\triangleright}=0, \quad E \cdot \mathbf{I}^{\triangleright}=0 .
\end{aligned}
$$

As the consequence, we obtain

$$
\begin{aligned}
\mathbf{I}_{\infty} & =\mathbf{e}_{\infty} \mathbf{I}_{\infty}^{\triangleright}=\mathbf{e}_{\infty} \wedge \mathbf{I}_{\infty}^{\triangleright}, \quad \mathbf{I}_{\infty} \wedge \mathbf{I}_{o}^{\triangleright}=\mathbf{e}_{\infty} \mathbf{I}^{\triangleright}=\mathbf{e}_{\infty} \wedge \mathbf{I}^{\triangleright}=\mathbf{I}^{\triangleright} \mathbf{e}_{\infty}, \\
3 \mathbf{I}_{o} & =\mathbf{e}_{o} \mathbf{I}_{o}^{\triangleright}=\mathbf{e}_{o} \wedge \mathbf{I}_{o}^{\triangleright}, \quad-3 \mathbf{I}_{\infty}^{\triangleright} \wedge \mathbf{I}_{o}=\mathbf{e}_{o} \mathbf{I}^{\triangleright}=\mathbf{e}_{o} \wedge \mathbf{I}^{\triangleright}=\mathbf{I}^{\triangleright} \mathbf{e}_{o}, \\
-3 \mathbf{I}_{\infty o} & =E \mathbf{I}^{\triangleright}=E \wedge \mathbf{I}^{\triangleright}=\mathbf{I}^{\triangleright} E .
\end{aligned}
$$

We can summarize the important set of relations

$$
\left\{1, \mathbf{e}_{o}, \mathbf{e}_{\infty}, E\right\} \wedge \mathbf{I}_{\infty}^{\triangleright} \wedge \mathbf{I}_{o}^{\triangleright}=\left\{1, \mathbf{e}_{o}, \mathbf{e}_{\infty}, E\right\} \mathbf{I}_{\infty}^{\triangleright} \wedge \mathbf{I}_{o}^{\triangleright}=\mathbf{I}_{\infty}^{\triangleright} \wedge \mathbf{I}_{o}^{\triangleright}\left\{1, \mathbf{e}_{o}, \mathbf{e}_{\infty}, E\right\} .
$$

We define the pseudo-scalar $\mathbf{I}_{\varepsilon}$ in $\mathbb{R}^{3}$ by

$$
\mathbf{I}_{\varepsilon}=\mathbf{e}_{1} \mathbf{e}_{2} \mathbf{e}_{3}, \quad \mathbf{I}_{\varepsilon}^{2}=-1, \quad \mathbf{I}_{\varepsilon}^{-1}=-\mathbf{I}_{\varepsilon},
$$

and the conformal pseudo-scalar $\mathbf{I}_{\mathrm{C}}$ in $\mathbb{R}^{4,1}$ by

$$
\mathbf{I}_{\mathrm{C}}=\mathbf{e}_{1} \mathbf{e}_{2} \mathbf{e}_{3} \mathbf{e}_{\infty} \wedge \mathbf{e}_{o}=\mathbf{I}_{\varepsilon} E, \quad \mathbf{I}_{\mathrm{C}}^{2}=-1, \quad \mathbf{I}_{\mathrm{C}}^{-1}=-\mathbf{I}_{\mathrm{C}},
$$

as well as the full 15-blade pseudo-scalar $\mathbf{I}$ of $\mathrm{Cl}(9,6)$ and its inverse $\mathbf{I}^{-1}$ (used for dualization $\mathbf{x} \rightarrow \mathbf{x}^{*}$ ):

$$
\mathbf{I}=\mathbf{I}_{\varepsilon} \mathbf{I}_{\infty o}=-\mathbf{I}_{\varepsilon} E_{1} E_{2} E_{3} E_{4} E_{5} E_{6}, \quad \mathbf{I}^{2}=-1, \quad \mathbf{I}^{-1}=-\mathbf{I} .
$$

The dual of a multivector indicates the division by the pseudo-scalar, e.g., $\mathbf{a}^{*}=-\mathbf{a I}$, $\mathbf{a}=\mathbf{a}^{*} \mathbf{I}$. From eq. (1.19) in [14], we have the useful duality between outer and inner products of non-scalar blades $A, B$ in geometric algebra:

$$
(A \wedge B)^{*}=A \cdot B^{*}, \quad A \wedge\left(B^{*}\right)=(A \cdot B)^{*} \Leftrightarrow A \wedge(B \mathbf{I})=(A \cdot B) \mathbf{I},
$$

which indicates that

$$
A \wedge B=0 \Leftrightarrow A \cdot B^{*}=0, \quad A \cdot B=0 \Leftrightarrow A \wedge B^{*}=0 .
$$

Using (2.23) and (2.24), useful duality relationships are

$$
\begin{aligned}
\left(\mathbf{I}_{\infty} \wedge \mathbf{I}_{o}\right)^{*} & =-\mathbf{I}_{\mathcal{E}}, \quad\left(\mathbf{I}_{\infty} \wedge \mathbf{I}_{o}^{\triangleright}\right)^{*}=-3 \mathbf{I}_{\varepsilon} \mathbf{e}_{\infty}, \\
\left(\mathbf{I}_{\varepsilon}\left(\mathbf{e}_{o i} \cdot \mathbf{I}_{\infty}\right) \wedge \mathbf{I}_{o}\right)^{*}=-\mathbf{e}_{o i}, & \left(\mathbf{I}_{\mathcal{E}} \mathbf{I}_{\infty} \wedge\left(\mathbf{e}_{\infty i} \cdot \mathbf{I}_{o}\right)\right)^{*}=-\mathbf{e}_{\infty i}, \\
\left(\mathbf{I}_{\varepsilon}\left(\mathbf{e}_{o} \cdot \mathbf{I}_{\infty}\right) \wedge \mathbf{I}_{o}\right)^{*}=-\mathbf{e}_{o}, & \left(\mathbf{I}_{\mathcal{\varepsilon}} \mathbf{I}_{\infty} \wedge\left(\mathbf{e}_{\infty} \cdot \mathbf{I}_{o}\right)\right)^{*}=-\mathbf{e}_{\infty} .
\end{aligned}
$$




\section{Points and embedded CGA objects in QCGA}

QCGA is an extension of conformal geometric algebra (CGA). Thus, objects defined in CGA are also defined in QCGA. The following sections introduce the important definition of a general point in QCGA, and show how all round and flat geometric objects (point pairs, flat points, circles, lines, spheres and planes) of CGA can be straightforwardly embedded in QCGA.

\subsection{Point in QCGA}

The point $\mathbf{x}$ of QCGA corresponding to the Euclidean point $\mathbf{x}_{\varepsilon}=x \mathbf{e}_{1}+y \mathbf{e}_{2}+z \mathbf{e}_{3} \in$ $\mathbb{R}^{3}$ is defined as

$$
\mathbf{x}=\mathbf{x}_{\varepsilon}+\frac{1}{2}\left(x^{2} \mathbf{e}_{\infty 1}+y^{2} \mathbf{e}_{\infty 2}+z^{2} \mathbf{e}_{\infty 3}\right)+x y \mathbf{e}_{\infty 4}+x z \mathbf{e}_{\infty 5}+y z \mathbf{e}_{\infty 6}+\mathbf{e}_{o} .
$$

Note that the null vectors $\mathbf{e}_{o 4}, \mathbf{e}_{o 5}, \mathbf{e}_{o 6}$ are not present in the definition of the point. This is merely to keep the convenient properties of the CGA points, namely, the inner product between two points is identical with the squared distance between them. Let $\mathbf{x}_{1}$ and $\mathbf{x}_{2}$ be two points. Then, their inner product is

$$
\begin{aligned}
& \mathbf{x}_{1} \cdot \mathbf{x}_{2}= \\
& \left(\mathbf{x}_{1 \varepsilon}+\frac{1}{2} x_{1}^{2} \mathbf{e}_{\infty 1}+\frac{1}{2} y_{1}^{2} \mathbf{e}_{\infty 2}+\frac{1}{2} z_{1}^{2} \mathbf{e}_{\infty 3}+x_{1} y_{1} \mathbf{e}_{\infty 4}+x_{1} z_{1} \mathbf{e}_{\infty 5}+y_{1} z_{1} \mathbf{e}_{\infty 6}+\mathbf{e}_{o}\right) \\
& \quad \cdot\left(\mathbf{x}_{2 \varepsilon}+\frac{1}{2} x_{2}^{2} \mathbf{e}_{\infty 1}+\frac{1}{2} y_{2}^{2} \mathbf{e}_{\infty 2}+\frac{1}{2} z_{2}^{2} \mathbf{e}_{\infty 3}+x_{2} y_{2} \mathbf{e}_{\infty 4}+x_{2} z_{2} \mathbf{e}_{\infty 5}+y_{2} z_{2} \mathbf{e}_{\infty 6}+\mathbf{e}_{o}\right) .
\end{aligned}
$$

from which together with Table 1, it follows that

$$
\mathbf{x}_{1} \cdot \mathbf{x}_{2}=\mathbf{x}_{1 \varepsilon} \cdot \mathbf{x}_{2 \varepsilon}-\frac{1}{2}\left(x_{1}^{2}+y_{1}^{2}+z_{1}^{2}+x_{2}^{2}+y_{2}^{2}+z_{2}^{2}\right)=-\frac{1}{2}\left(\mathbf{x}_{1 \varepsilon}-\mathbf{x}_{2 \varepsilon}\right)^{2} .
$$

We see that the inner product is equivalent to minus half of the squared Euclidean distance between the two points $\mathbf{x}_{1}$ and $\mathbf{x}_{2}$.

In the remainder of this paper, the following result will be useful, because it relates a point in QCGA to the representation in CGA $\mathbb{R}^{4,1}$ with vector basis $\left\{\mathbf{e}_{o}, \mathbf{e}_{1}, \mathbf{e}_{2}, \mathbf{e}_{3}, \mathbf{e}_{\infty}\right\}$.

$$
\begin{aligned}
\mathbf{x} \wedge \mathbf{I}_{\infty}^{\triangleright} & =\left(\mathbf{x}_{\varepsilon}+\frac{1}{2}\left(x^{2} \mathbf{e}_{\infty 1}+y^{2} \mathbf{e}_{\infty 2}+z^{2} \mathbf{e}_{\infty 3}\right)+\mathbf{e}_{o}\right) \wedge \mathbf{I}_{\infty}^{\triangleright} \\
& =\left(\mathbf{x}_{\varepsilon}+\mathbf{e}_{o}\right) \wedge \mathbf{I}_{\infty}^{\triangleright}+\frac{1}{2}\left(x^{2} \mathbf{e}_{\infty 1}+y^{2} \mathbf{e}_{\infty 2}+z^{2} \mathbf{e}_{\infty 3}\right) \wedge \mathbf{I}_{\infty}^{\triangleright} \\
& =\left(\mathbf{x}_{\varepsilon}+\mathbf{e}_{o}\right) \wedge \mathbf{I}_{\infty}^{\triangleright}+\frac{1}{2}\left(x^{2}+y^{2}+z^{2}\right) \mathbf{e}_{\infty} \wedge \mathbf{I}_{\infty}^{\triangleright} \\
& =\left(\mathbf{x}_{\varepsilon}+\mathbf{e}_{o}\right) \wedge \mathbf{I}_{\infty}^{\triangleright}+\frac{1}{2} \mathbf{x}_{\varepsilon}^{2} \mathbf{e}_{\infty} \wedge \mathbf{I}_{\infty}^{\triangleright} \\
& =\left(\mathbf{x}_{\varepsilon}+\frac{1}{2} \mathbf{x}_{\varepsilon}^{2} \mathbf{e}_{\infty}+\mathbf{e}_{o}\right) \wedge \mathbf{I}_{\infty}^{\triangleright}=\mathbf{x}_{C} \wedge \mathbf{I}_{\infty}^{\triangleright}=\mathbf{x}_{C} \mathbf{I}_{\infty}^{\triangleright},
\end{aligned}
$$

where we have dropped in the first line the cross terms $x y \mathbf{e}_{\infty 4}+x z \mathbf{e}_{\infty 5}+y z \mathbf{e}_{\infty 6}$, because wedging with $\mathbf{I}_{\infty b}=\mathbf{e}_{\infty 4} \wedge \mathbf{e}_{\infty 5} \wedge \mathbf{e}_{\infty 6}$, a factor in $\mathbf{I}_{\infty}^{\triangleright}=\mathbf{I}_{\infty a}^{\triangleright} \mathbf{I}_{\infty b}$, eliminates them. Therefore, if a point in QCGA appears wedged with $\mathbf{I}_{\infty}^{\triangleright}$, we can always replace it by the form

$$
\mathbf{x}_{\mathrm{C}}=\mathbf{x}_{\varepsilon}+\frac{1}{2} \mathbf{x}_{\varepsilon}^{2} \mathbf{e}_{\infty}+\mathbf{e}_{o}=-\frac{1}{3} \mathbf{x} \wedge \mathbf{I}_{\infty}^{\triangleright} \mid \mathbf{I}_{o}^{\triangleright} .
$$

This, in turn, means that we can embed in QCGA the known CGA representations [14] in $C l(4,1)$ of round and flat objects, by taking the outer products of between one and five points with $\mathbf{I}_{\infty}^{\triangleright}$, as further shown below. 


\subsection{Round and flat objects in QCGA}

We refer points, point pairs, circles, and spheres with uniform curvature as round objects. Similar to CGA, these can be defined by the outer product of one to four points with $\mathbf{I}_{\infty}^{\triangleright}$. Their centre $\mathbf{c}_{C}$, radius $r$ and Euclidean carrier $D$ can be easily extracted. Moreover, they can be directly constructed from their centre $\mathbf{c}_{\mathrm{C}}$, radius $r$ and Euclidean carrier $D$.

Wedging any round object with the point at infinity $\mathbf{e}_{\infty}$, gives the corresponding flat object multivector. From it the orthogonal distance to the origin $\mathbf{c}_{\varepsilon \perp}$ and the Euclidean carrier $D$ can easily be extracted.

We now briefly review the CGA description of round and flat objects embedded in QCGA. The round objects are

$$
\begin{aligned}
P & =\mathbf{x} \wedge \mathbf{I}_{\infty}^{\triangleright}=\mathbf{x}_{\mathrm{C}} \mathbf{I}_{\infty}^{\triangleright}, \\
P p & =\mathbf{x}_{1} \wedge \mathbf{x}_{2} \wedge \mathbf{I}_{\infty}^{\triangleright}=\mathbf{x}_{1 \mathrm{C}} \wedge \mathbf{x}_{2 \mathrm{C}} \mathbf{I}_{\infty}^{\triangleright}, \\
\text { Circle } & =\mathbf{x}_{1} \wedge \mathbf{x}_{2} \wedge \mathbf{x}_{3} \wedge \mathbf{I}_{\infty}^{\triangleright}=\mathbf{x}_{1 \mathrm{C}} \wedge \mathbf{x}_{2 \mathrm{C}} \wedge \mathbf{x}_{3 \mathrm{C}} \mathbf{I}_{\infty}^{\triangleright}, \\
\text { Sphere } & =\mathbf{x}_{1} \wedge \mathbf{x}_{2} \wedge \mathbf{x}_{3} \wedge \mathbf{x}_{4} \wedge \mathbf{I}_{\infty}^{\triangleright}=\mathbf{x}_{1 \mathrm{C}} \wedge \mathbf{x}_{2 \mathrm{C}} \wedge \mathbf{x}_{3 \mathrm{C}} \wedge \mathbf{x}_{4 \mathrm{C}} \mathbf{I}_{\infty}^{\triangleright} .
\end{aligned}
$$

The corresponding flat objects are

$$
\begin{aligned}
\text { Flatp } & =-P \wedge \mathbf{e}_{\infty}=\mathbf{x} \wedge \mathbf{e}_{\infty} \wedge \mathbf{I}_{\infty}^{\triangleright}=\mathbf{x}_{\mathrm{C}} \wedge \mathbf{e}_{\infty} \mathbf{I}_{\infty}^{\triangleright}, \\
\text { Line } & =-P p \wedge \mathbf{e}_{\infty}=\mathbf{x}_{1} \wedge \mathbf{x}_{2} \wedge \mathbf{e}_{\infty} \wedge \mathbf{I}_{\infty}^{\triangleright}=\mathbf{x}_{1} \wedge \mathbf{x}_{2} \wedge \mathbf{e}_{\infty} \wedge \mathbf{I}_{\infty} \\
& =\mathbf{x}_{1 \mathrm{C}} \wedge \mathbf{x}_{2 \mathrm{C}} \wedge \mathbf{e}_{\infty} \mathbf{I}_{\infty}^{\triangleright}, \\
\text { Plane } & =- \text { Circle } \wedge \mathbf{e}_{\infty}=\mathbf{x}_{1} \wedge \mathbf{x}_{2} \wedge \mathbf{x}_{3} \wedge \mathbf{e}_{\infty} \wedge \mathbf{I}_{\infty}^{\triangleright}=\mathbf{x}_{1 \mathrm{C}} \wedge \mathbf{x}_{2 \mathrm{C}} \wedge \mathbf{x}_{3 \mathrm{C}} \wedge \mathbf{e}_{\infty} \mathbf{I}_{\infty}^{\triangleright}, \\
\text { Space } & =- \text { Sphere } \wedge \mathbf{e}_{\infty}=\mathbf{x}_{1} \wedge \mathbf{x}_{2} \wedge \mathbf{x}_{3} \wedge \mathbf{x}_{4} \wedge \mathbf{e}_{\infty} \wedge \mathbf{I}_{\infty}^{\triangleright} \\
& =\mathbf{x}_{1 \mathrm{C}} \wedge \mathbf{x}_{2 \mathrm{C}} \wedge \mathbf{x}_{3 \mathrm{C}} \wedge \mathbf{x}_{4 \mathrm{C}} \wedge \mathbf{e}_{\infty} \mathbf{I}_{\infty}^{\triangleright} .
\end{aligned}
$$

The above embeddings by means of the outer product with $\mathbf{I}_{\infty}^{\triangleright}$, allow to use standard CGA results found in [14]. All embedded round entities of point, point pair, circle, and sphere (spheres in zero, one, two and three dimensions) have one common multivector form ${ }^{2}$

$$
\begin{aligned}
S & =\left(D \wedge \mathbf{c}_{\varepsilon}+\left[\frac{1}{2}\left(\mathbf{c}_{\varepsilon}^{2}+r^{2}\right) D-\mathbf{c}_{\varepsilon} \mathbf{c}_{\varepsilon}\right\rfloor D\right] \mathbf{e}_{\infty}+D \mathbf{e}_{o}+D\left\lfloor\mathbf{c}_{\varepsilon} E\right) \mathbf{I}_{\infty}^{\triangleright}=S_{\mathrm{C}} \mathbf{I}_{\infty}^{\triangleright}, \\
S_{\mathrm{C}} & =-\frac{1}{3} S\left\lfloor\mathbf{I}_{o}^{\triangleright} .\right.
\end{aligned}
$$

The Euclidean carriers $D$ are for each object are Euclidean scalar, vector, bivector and trivector, respectively,

$$
D=\left\{\begin{aligned}
1, & \text { point } \mathbf{x} \\
\mathbf{d}_{\varepsilon}, & \text { point pair } P p \\
\mathbf{i}_{c}, & \text { circle Circle } \\
\mathbf{I}_{\varepsilon}, & \text { sphere Sphere }
\end{aligned}\right.
$$

where the unit point pair connection direction vector is $\mathbf{d}_{\varepsilon}=\left(\mathbf{x}_{1 \varepsilon}-\mathbf{x}_{2 \varepsilon}\right) / 2 r$ and the Euclidean circle plane bivector is $\mathbf{i}_{c}$. The radius $r$ of a round object and its centre $\mathbf{c}_{\mathrm{C}}$

${ }^{2}$ Note that the product symbols $\rfloor$ and $\lfloor$ express left- and right contraction, respectively. 
are generally determined by

$$
r^{2}=\frac{S_{\mathrm{C}} \widetilde{S_{\mathrm{C}}}}{\left(S_{\mathrm{C}} \wedge \mathbf{e}_{\infty}\right)\left(S_{\mathrm{C}} \wedge \mathbf{e}_{\infty}\right)^{2}}, \quad \mathbf{c}_{\mathrm{C}}=S_{\mathrm{C}} \mathbf{e}_{\infty} S_{\mathrm{C}},
$$

where the tilde symbol indicates the reverse operator.

All embedded flat entities of flat point, line, plane, and space have one common multivector form

$$
\begin{aligned}
F & =-S \wedge \mathbf{e}_{\infty}=\left(D \wedge \mathbf{c}_{\varepsilon} \mathbf{e}_{\infty}-D E\right) \mathbf{I}_{\infty}^{\triangleright}=\left(D \mathbf{c}_{\varepsilon \perp} \mathbf{e}_{\infty}-D E\right) \mathbf{I}_{\infty}^{\triangleright}=F_{\mathrm{C}} \mathbf{I}_{\infty}^{\triangleright}, \\
F_{\mathrm{C}} & =-S_{\mathrm{C}} \wedge \mathbf{e}_{\infty}=\frac{1}{3} F\left\lfloor\mathbf{I}_{o}^{\triangleright},\right.
\end{aligned}
$$

where the orthogonal Euclidean distance of the flat object from the origin is

$$
\mathbf{c}_{\varepsilon \perp}=\left\{\begin{array}{rl}
\mathbf{x}_{\varepsilon}, & \text { finite-infinite point pair Flatp } \\
\mathbf{c}_{\varepsilon \perp}, & \text { line Line } \\
\mathbf{c}_{\varepsilon \perp}, & \text { plane Plane } \\
0, & \text { 3D space Space }
\end{array} .\right.
$$

The Euclidean carrier blade $D$, and the orthogonal Euclidean distance vector of $F$ from the origin, can both be directly determined from the flat object multivector as

$$
D=F_{\mathrm{C}}\left\lfloor E, \quad \mathbf{c}_{\varepsilon \perp}=D^{-1}\left(F_{\mathrm{C}} \wedge \mathbf{e}_{o}\right)\lfloor E .\right.
$$

For a further detailed description of lines, planes and spheres in QCGA, we refer to [2].

\section{Quadratic surfaces from contact points}

This section describes how QCGA handles quadratic surfaces. All the embedded CGA objects in QCGA defined in Section 3 are thus a part of a more general framework algebraic.

A quadratic surface in $\mathbb{R}^{3}$ is implicitly formulated as

$$
F(x, y, z)=\mathrm{a} x^{2}+\mathrm{b} y^{2}+\mathrm{c} z^{2}+\mathrm{d} x y+\mathrm{e} x z+\mathrm{f} y z+\mathrm{g} x+\mathrm{h} y+\mathrm{i} z+\mathrm{j}=0 .
$$

A quadratic surface is constructed by the outer product of nine contact points as follows

$$
\mathbf{q}=\mathbf{x}_{1} \wedge \mathbf{x}_{2} \wedge \cdots \wedge \mathbf{x}_{9}
$$

Note that in [2], the definition of $\mathbf{q}$ additionally included wedging with $\mathbf{I}_{o}^{\triangleright}$ (thus forming a pseudovector of grade 14 in $C l(9,6))$, but as found in [13], this would seriously impede the use of versor operators for geometric transformations of rotation, translation and scaling, due to the lack of transformation invariance of $\mathbf{I}_{o}^{\triangleright}$. The multivector $\mathbf{q}$ corresponds to the primal form of a quadratic surface in QCGA, with grade nine and twelve components. Again three of these components have the same coefficient and can be combined together in a form defined by only ten coefficients $a, b, \ldots, j$, and we obtain a quadratic surface $\mathbf{q}$ and the related computationally 
efficient dual vector of $\left(\mathbf{q} \wedge \mathbf{I}_{o}^{\triangleright}\right)^{*}$ as

$$
\begin{aligned}
& \mathbf{q} \wedge \mathbf{I}_{o}^{\triangleright}=\mathbf{I}_{\varepsilon}\left(\left(2 \mathrm{a} \mathbf{e}_{o 1}+2 \mathrm{~b} \mathbf{e}_{o 2}+2 \mathrm{c} \mathbf{e}_{o 3}+\mathrm{d} \mathbf{e}_{o 4}+\mathrm{e} \mathbf{e}_{o 5}+\mathrm{f} \mathbf{e}_{o 6}\right) \cdot \mathbf{I}_{\infty}\right) \wedge \mathbf{I}_{o} \\
& +\left(\mathrm{ge}_{1}+\mathrm{h} \mathbf{e}_{2}+\mathrm{i} \mathbf{e}_{3}\right) \mathbf{I}_{\varepsilon} \mathbf{I}_{\infty O}+\mathrm{j} \mathbf{I}_{\varepsilon} \mathbf{I}_{\infty} \wedge\left(\mathbf{e}_{\infty} \cdot \mathbf{I}_{o}\right) \\
& =\left(-\left(2 \mathrm{ae}_{o 1}+2 \mathrm{~b} \mathbf{e}_{o 2}+2 \mathrm{c} \mathbf{e}_{o 3}+\mathrm{d} \mathbf{e}_{o 4}+\mathrm{e} \mathbf{e}_{o 5}+\mathrm{f} \mathbf{e}_{o 6}\right)\right. \\
& \left.+\left(\mathrm{ge}_{1}+\mathrm{h} \mathbf{e}_{2}+\mathrm{i} \mathbf{e}_{3}\right)-\mathrm{je} \mathbf{e}_{\infty}\right) \mathbf{I} \\
& =\left(\mathbf{q} \wedge \mathbf{I}_{o}^{\triangleright}\right)^{*} \mathbf{I},
\end{aligned}
$$

where in the second equality we used the duality relationships of (2.40). The expression for the dual vector $\left(\mathbf{q} \wedge \mathbf{I}_{o}^{\triangleright}\right)^{*}$ is therefore

$$
\begin{gathered}
\left(\mathbf{q} \wedge \mathbf{I}_{o}^{\triangleright}\right)^{*}=-\left(2 \mathrm{ae}_{o 1}+2 \mathrm{~b} \mathbf{e}_{o 2}+2 \mathrm{c} \mathbf{e}_{o 3}+\mathrm{de} \mathbf{e}_{o 4}+\mathrm{e} \mathbf{e}_{o 5}+\mathrm{f} \mathbf{e}_{o 6}\right) \\
+\left(\mathrm{ge}_{1}+\mathrm{he}_{2}+\mathrm{ie}_{3}\right)-\mathrm{j} \mathbf{e}_{o} .
\end{gathered}
$$

Proposition 4.1. A point $\mathbf{x}$ lies on the quadratic surface $\mathbf{q}$, if and only if $\mathbf{x} \wedge \mathbf{q} \wedge \mathbf{I}_{o}^{\triangleright}=$ 0 .

Proof.

$$
\begin{aligned}
\mathbf{x} \wedge\left(\mathbf{q} \wedge \mathbf{I}_{o}^{\triangleright}\right)= & \mathbf{x} \wedge\left(\left(\mathbf{q} \wedge \mathbf{I}_{o}^{\triangleright}\right)^{*} \mathbf{I}\right)=\mathbf{x} \cdot\left(\mathbf{q} \wedge \mathbf{I}_{o}^{\triangleright}\right)^{*} \mathbf{I} \\
= & \mathbf{x} \cdot\left(-\left(2 \mathrm{ae}_{o 1}+2 \mathrm{~b} \mathbf{e}_{o 2}+2 \mathrm{c} \mathbf{e}_{o 3}+\mathrm{d} \mathbf{e}_{o 4}+\mathrm{e} \mathbf{e}_{o 5}+\mathrm{f} \mathbf{e}_{o 6}\right)\right. \\
& \left.\quad+\left(\mathrm{ge}_{1}+\mathrm{h} \mathbf{e}_{2}+\mathrm{i} \mathbf{e}_{3}\right)-\mathrm{j} \mathbf{e}_{\infty}\right) \mathbf{I} \\
= & \left(\mathrm{a} x^{2}+\mathrm{b} y^{2}+\mathrm{c} z^{2}+\mathrm{d} x y+\mathrm{e} x z+\mathrm{f} y z+\mathrm{g} x+\mathrm{h} y+\mathrm{i} z+\mathrm{j}\right) \mathbf{I} .
\end{aligned}
$$

This corresponds to the implicit formula (4.1) representing a general quadratic surface.

The dualization of the primal quadratic surface (4.2) wedged with $\mathbf{I}_{o}^{\triangleright}$ leads to the dual 1-vector $\left(\mathbf{q} \wedge \mathbf{I}_{o}^{\triangleright}\right)^{*}$ of (4.4). Dualization of (4.5) gives us the following corollary.

Corollary 4.2. A point $\mathbf{x}$ lies on the dual quadratic surface $\mathbf{q}^{*}$ if and only if $\mathbf{x} \cdot(\mathbf{q} \wedge$ $\left.\mathbf{I}_{o}^{\triangleright}\right)^{*}=0$.

\section{Aligned quadratic surfaces from contact points}

Up to now, we defined general quadratic surfaces using the outer product of nine points. For simplicity purpose, one might sometimes prefer to define axis-aligned quadratic surfaces from fewer points. The implicit equation of an axis-aligned quadratic surface is as follows:

$$
F(x, y, z)=\mathrm{a} x^{2}+\mathrm{b} y^{2}+\mathrm{c} z^{2}+\mathrm{g} x+\mathrm{h} y+\mathrm{i} z+\mathrm{j}=0 .
$$

On one hand, this equation has seven coefficients and six degrees of freedom. An axis-aligned quadratic surface can then be constructed by computing the outer product of six points. On the other hand, one has to remove the cross terms $x y, x z, y z$ in the representation of points to be able to satisfy equation (5.1). To achieve this, 
our solution is to compute the outer product with $\mathbf{e}_{\infty 4}, \mathbf{e}_{\infty 5}, \mathbf{e}_{\infty 6}$, i.e. with $\mathbf{I}_{\infty b}=\mathbf{e}_{\infty 4} \wedge$ $\mathbf{e}_{\infty 5} \wedge \mathbf{e}_{\infty 6}$. Indeed, one finds that any point $\mathbf{x}$ satisfies

$$
\mathbf{x} \wedge \mathbf{e}_{\infty 4} \wedge \mathbf{e}_{\infty 5} \wedge \mathbf{e}_{\infty 6}=\mathbf{x} \wedge \mathbf{I}_{\infty b}=\left(\mathbf{e}_{o}+\mathbf{x}_{\varepsilon}+\frac{1}{2}\left(x^{2} \mathbf{e}_{\infty 1}+y^{2} \mathbf{e}_{\infty 2}+z^{2} \mathbf{e}_{\infty 3}\right)\right) \wedge \mathbf{I}_{\infty b} .
$$

Thus, one can consider that an axis aligned quadratic surface is a general quadratic surface where three points are sent to infinity $\mathbf{e}_{\infty 4}, \mathbf{e}_{\infty 5}, \mathbf{e}_{\infty 6}$ in the following way:

$$
\mathbf{q}=\mathbf{x}_{1} \wedge \mathbf{x}_{2} \wedge \mathbf{x}_{3} \wedge \mathbf{x}_{4} \wedge \mathbf{x}_{5} \wedge \mathbf{x}_{6} \wedge \mathbf{I}_{\infty b} .
$$

This grade nine multivector blade $\mathbf{q}$ corresponds to the primal form of a quadratic surface, outer product of six points with three $\mathbf{e}_{\infty i}, i=4,5,6$, basis vector factors in $\mathbf{I}_{\infty b}$, and this quadratic surface has nine components. For the same reason as in the construction of the general quadratic surface, we can combine three components having the same coefficient. Furthermore, computing the outer product with $\mathbf{I}_{\infty b}=$ $\mathbf{e}_{\infty 4} \wedge \mathbf{e}_{\infty 5} \wedge \mathbf{e}_{\infty 6}$ removes the components $\mathbf{e}_{\infty 4}, \mathbf{e}_{\infty 5}, \mathbf{e}_{\infty 6}$ of each of the six points. Combining the outer product of such points with null basis vectors and wedging with $\mathbf{I}_{o}^{\triangleright}$, results in the form defined by the seven coefficients a, b, c, g, h, i, j as

$$
\begin{aligned}
\mathbf{q} \wedge \mathbf{I}_{o}^{\triangleright}=\quad \mathbf{I}_{\varepsilon}( & \left.\left(2 \mathrm{ae}_{o 1}+2 \mathrm{~b} \mathbf{e}_{o 2}+2 \mathrm{c} \mathbf{e}_{o 3}\right) \cdot \mathbf{I}_{\infty}\right) \wedge \mathbf{I}_{o} \\
& +\left(\mathrm{ge}_{1}+\mathrm{he}_{2}+\mathrm{ie}_{3}\right) \mathbf{I}_{\varepsilon} \mathbf{I}_{\infty} \wedge \mathbf{I}_{o}+\mathrm{j} \mathbf{I}_{\infty} \wedge\left(\mathbf{e}_{\infty} \cdot \mathbf{I}_{o}\right) .
\end{aligned}
$$

Proposition 5.1. A point $\mathbf{x}$ lies on an axis-aligned quadratic surface $\mathbf{q}$, iff $\mathbf{x} \wedge \mathbf{q} \wedge$ $\mathbf{I}_{o}^{\triangleright}=0$.

Proof.

$$
\begin{aligned}
\mathbf{x} \wedge \mathbf{q} \wedge \mathbf{I}_{o}^{\triangleright} & =\mathbf{x} \wedge\left(\left(\mathbf{q} \wedge \mathbf{I}_{o}^{\triangleright}\right)^{*} \mathbf{I}\right)=\mathbf{x} \cdot\left(\mathbf{q} \wedge \mathbf{I}_{o}^{\triangleright}\right)^{*} \mathbf{I} \\
& =\left(\mathbf{x} \cdot\left(-2\left(\mathrm{ae}_{o 1}+\mathrm{b} \mathbf{e}_{o 2}+\mathrm{c} \mathbf{e}_{o 3}\right)+\mathrm{g} \mathbf{e}_{1}+\mathrm{h} \mathbf{e}_{2}+\mathrm{i} \mathbf{e}_{3}-\mathrm{j} \mathbf{e}_{\infty}\right)\right) \mathbf{I} \\
& =\left(\mathrm{a} x^{2}+\mathrm{b} y^{2}+\mathrm{c} z^{2}+\mathrm{g} x+\mathrm{h} y+\mathrm{i} z+\mathrm{j}\right) \mathbf{I} .
\end{aligned}
$$

This corresponds to the formula (5.1) representing an axis-aligned quadratic surface.

Now it is easy to construct an axis-aligned quadratic surface by properly choosing the contact points that lie on the chosen axis-aligned quadratic surface. The next sections present some examples of chosen axis-aligned quadratic surfaces, with some chosen points that lie on these quadratic surfaces.

\subsection{Representation of a primal axis-aligned paraboloid}

We can construct the axis-aligned elliptic paraboloid using six points that lie on it. For example, the points:

$$
\begin{array}{lll}
\mathbf{x}_{1}(0.0,0.0,0.0), & \mathbf{x}_{2}(-0.39,0.1,0.33), & \mathbf{x}_{3}(0.0,-0.41,0.5), \\
\mathbf{x}_{4}(0.0,0.23,0.17), & \mathbf{x}_{5}(0.47,0.0,0.45), & \mathbf{x}_{6}(0.29,-0.27,0.4),
\end{array}
$$

lie on an axis-aligned elliptic paraboloid. The result of equation (5.3) applied to these points is shown in Figure 1. 
3D quadrics in extended CGA, control points, implicit equations, alignment 11

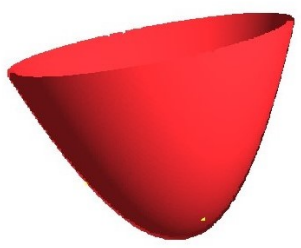

FIGURE 1. Result of one paraboloid from six points.

\subsection{Representation of a primal axis-aligned hyperbolic paraboloid}

Using the same equation, and replacing the contact points by some that lie on an axis-aligned hyperbolic paraboloid

$$
\begin{array}{lll}
\mathbf{x}_{1}(0.0,0.0,0.0), & \mathbf{x}_{2}(0.45,-0.01,0.2), & \mathbf{x}_{3}(0.34,-0.37,-0.17), \\
\mathbf{x}_{4}(-0.47,-0.18,0.15), & \mathbf{x}_{5}(-0.36,0.12,0.1), & \mathbf{x}_{6}(0.18,0.13,0.0),
\end{array}
$$

results in the axis-aligned hyperbolic paraboloid shown in Figure 2.

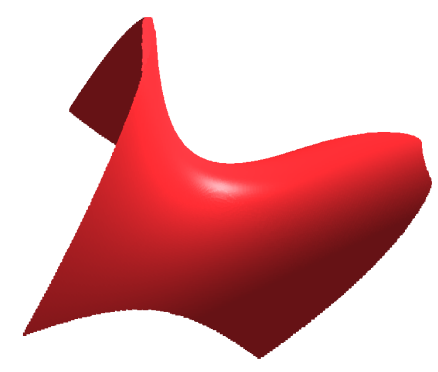

FIGURE 2. Result of one hyperbolic paraboloid from six points.

\subsection{Representation of a primal axis-aligned cylinder}

An axis-aligned cylinder is an axis-aligned quadratic surface where one of the squared components is removed with respect to the axis of the cylinder.

On one hand, this supposes that an axis-aligned cylinder can be constructed using only five points. On the other hand, this means that the considered component of each point taken to construct the quadratic surface has to be removed. In a QCGA point, the squared components lie in the $\mathbf{e}_{\infty 1}, \mathbf{e}_{\infty 2}, \mathbf{e}_{\infty 3}$ components. Thus, replacing one point in equation (5.3) by a point at infinity with respect to the desired alignment axis (choosing from $\left\{\mathbf{e}_{\infty 1}, \mathbf{e}_{\infty 2}, \mathbf{e}_{\infty 3}\right\}$ for $x$-, $y$-, or $z$-axis alignment, respectively) defines the desired axis-aligned cylinder. For example, one can define an axis-aligned 
cylinder along the $z$-axis from only five points. Therefore, in equation (5.3), we can replace a point by one of the points at infinity, i.e., $\mathbf{e}_{\infty 3}$ for a $z$-axis aligned centred cylinder. For example, we choose the following five points

$$
\begin{gathered}
\mathbf{x}_{1}(-0.2,0.1,0.3), \quad \mathbf{x}_{2}(0.4,0.1,0.2), \quad \mathbf{x}_{3}(0.1,0.4,0.1), \\
\mathbf{x}_{4}(0.1,-0.2,0.4), \quad \mathbf{x}_{5}(0.1,-0.2,-0.4),
\end{gathered}
$$

and the outer product between these five points and $\mathbf{e}_{\infty 3}$ with $\mathbf{I}_{\infty b}$ (for axis alignment) as

$$
\mathbf{q}=\mathbf{x}_{1} \wedge \mathbf{x}_{2} \wedge \mathbf{x}_{3} \wedge \mathbf{x}_{4} \wedge \mathbf{x}_{5} \wedge \mathbf{e}_{\infty 3} \wedge \mathbf{I}_{\infty b}
$$

The cylinder whose axis is the (Oy) axis can be constructed by replacing $\mathbf{e}_{\infty 3}$ in the above equation by $\mathbf{e}_{\infty 2}$ :

$$
\mathbf{q}=\mathbf{x}_{1} \wedge \mathbf{x}_{2} \wedge \mathbf{x}_{3} \wedge \mathbf{x}_{4} \wedge \mathbf{x}_{5} \wedge \mathbf{e}_{\infty 2} \wedge \mathbf{I}_{\infty b}
$$

And finally, the cylinder whose axis is the $(\mathrm{Ox})$ axis is obtained by replacing $\mathbf{e}_{\infty 3}$ by $\mathbf{e}_{\infty 1}$ :

$$
\mathbf{q}=\mathbf{x}_{1} \wedge \mathbf{x}_{2} \wedge \mathbf{x}_{3} \wedge \mathbf{x}_{4} \wedge \mathbf{x}_{5} \wedge \mathbf{e}_{\infty 1} \wedge \mathbf{I}_{\infty b}
$$

Figure 3 shows three cylinders: one along $(O x)$, another along $(O y)$, and the third one along $(\mathrm{Oz})$.

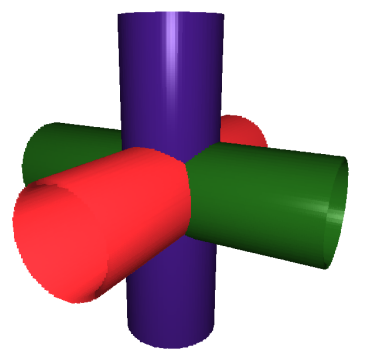

FIgURE 3. Construction of three cylinders along $(O x)$ in blue, $(O y)$ in green and $O z)$ in red, applying (5.8), (5.7) and (5.6). Each cylinder is constructed from five points and has the same diameter.

\subsection{Representation of a primal axis-aligned elliptic cylinder}

As five points are enough to uniquely define a cylinder, five points define also an axis-aligned elliptic cylinder whose main axis is given by the null basis vector replacing the sixth point. For example, an axis-aligned elliptic cylinder whose main axis is $(O z)$ can be defined with the following five points lying on it

$$
\begin{gathered}
\mathbf{x}_{1}(-0.44,0.0,0.0), \quad \mathbf{x}_{2}(0.0,-0.2,0.0), \quad \mathbf{x}_{3}(0.3,0.15,0.15), \\
\mathbf{x}_{4}(0.0,0.2,0.3), \quad \mathbf{x}_{5}(0.44,0.0,0.4) .
\end{gathered}
$$

The result is represented in Figure 4. 


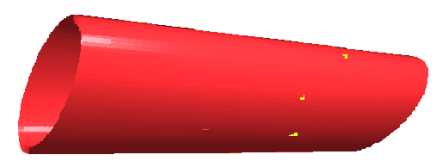

FIGURE 4. Construction of one elliptic cylinder from five points.

\subsection{Representation of a primal axis-aligned spheroid}

A spheroid is characterized as an ellipsoid having two of its axes whose length is equal. Again, this property supposes that an axis-aligned spheroid can be constructed from five points. Furthermore, one has to constrain each point such that the squared components along the two of its axes have the same length. This is achieved by the outer product of the points and the vector $\mathbf{e}_{\infty i}-\mathbf{e}_{\infty j}$, where $i$ and $j \neq i$, specify the two equal length axes. This 1-vector $\mathbf{e}_{\infty i}-\mathbf{e}_{\infty j}$ can be geometrically seen as the bisecting plane at infinity along the two considered axes, leading to some new geometric interpretations in the algebra.

Further geometric understanding of the algebraic operation of the outer product with $\mathbf{e}_{\infty i}-\mathbf{e}_{\infty j}, i$ and $j \neq i$, can be gained from expanding the five blade $\mathbf{I}_{\infty}^{\triangleright}$, that is instrumental for embedding CGA objects in QCGA, as explained in Section 3.2. The expansion gives

$$
\begin{aligned}
\mathbf{I}_{\infty}^{\triangleright} & =\mathbf{I}_{\infty a}^{\triangleright} \wedge \mathbf{I}_{\infty b}=\left(\mathbf{e}_{\infty 1}-\mathbf{e}_{\infty 2}\right) \wedge\left(\mathbf{e}_{\infty 2}-\mathbf{e}_{\infty 3}\right) \wedge \mathbf{I}_{\infty b} \\
& =\left(\mathbf{e}_{\infty 2}-\mathbf{e}_{\infty 3}\right) \wedge\left(\mathbf{e}_{\infty 3}-\mathbf{e}_{\infty 1}\right) \wedge \mathbf{I}_{\infty b} \\
& =\left(\mathbf{e}_{\infty 1} \wedge \mathbf{e}_{\infty 2}+\mathbf{e}_{\infty 2} \wedge \mathbf{e}_{\infty 3}+\mathbf{e}_{\infty 3} \wedge \mathbf{e}_{\infty 1}\right) \wedge \mathbf{I}_{\infty b},
\end{aligned}
$$

where we see that the three factors $\mathbf{e}_{\infty 1}-\mathbf{e}_{\infty 2}, \mathbf{e}_{\infty 2}-\mathbf{e}_{\infty 3}$ and $\mathbf{e}_{\infty 3}-\mathbf{e}_{\infty 1}$, are all factors of $\mathbf{I}_{\infty}^{\triangleright}$, thus producing circles in every coordinate plane and in every dimension, and the second trivector blade factor $\mathbf{I}_{\infty b}$ forces axis alignment, as discussed at the beginning of the current section. In that sense we understand that wedging four points in (3.9) with $\mathbf{I}_{\infty}^{\triangleright}$, necessarily leads to a sphere with circular cross sections in every coordinate plane, and with axis alignment, even though this latter fact is subtle for isotropic objects, like spheres. In CGA a plane is the limiting case of a sphere with infinite radius. The other objects of circle and line, point pair and flat point, are simply the lower dimensional versions of the spherical and planar case in three dimensions.

Thus, we can construct a spheroid having equal length axis $O x$ and $O y$ by

$$
\mathbf{x}_{1} \wedge \mathbf{x}_{2} \wedge \mathbf{x}_{3} \wedge \mathbf{x}_{4} \wedge \mathbf{x}_{5} \wedge\left(\mathbf{e}_{\infty 1}-\mathbf{e}_{\infty 2}\right) \wedge \mathbf{I}_{\infty b}
$$


Note that the sixth point is replaced by $\left(\mathbf{e}_{\infty 1}-\mathbf{e}_{\infty 2}\right)$. As an example, we construct the axis-aligned prolate (elongated in the $z$-axis) spheroid passing through the five following points lying on a prolate spheroid

$$
\begin{gathered}
\mathbf{x}_{1}(-0.26,0.0,0.0), \quad \mathbf{x}_{2}(0.03,0.22,0.24), \quad \mathbf{x}_{3}(-0.2,-0.1,-0.23), \\
\mathbf{x}_{4}(0.0,0.26,0.0), \quad \mathbf{x}_{5}(0.0,0.0,0.45) .
\end{gathered}
$$

The resulting surface is shown in Figure 5.

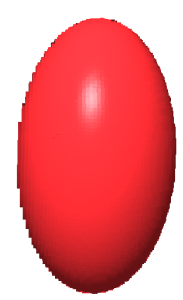

FIGURE 5. Construction of axis-aligned prolate spheroid from five points.

\subsection{Representation of a primal axis-aligned pair of planes}

Sending one point of a sphere to infinity $\mathbf{e}_{\infty}$ results in the plane passing through the remaining points. Now by sending two points of an ellipsoid to infinity, we obtain a pair of parallel planes. This indicates that an axis-aligned pair of planes can be constructed from four points. Then, this can be achieved by the outer product of four points $\mathbf{x}_{1}, \mathbf{x}_{2}, \mathbf{x}_{3}, \mathbf{x}_{4}$ and two points at infinity $\mathbf{e}_{\infty 1}, \mathbf{e}_{\infty 2}$ in the following equation

$$
\mathbf{x}_{1} \wedge \mathbf{x}_{2} \wedge \mathbf{x}_{3} \wedge \mathbf{x}_{4} \wedge \mathbf{e}_{\infty 1} \wedge \mathbf{e}_{\infty 2} \wedge \mathbf{I}_{\infty b}
$$

\subsection{Representation of a primal axis-aligned curve}

Two points define a bi-cylindrical curve, meaning that the curve obtained by the intersection of two cylinders. Therefore, given two points and three points at infinity, it is possible to construct a bi-cylindrical curve as follows

$$
\mathbf{x}_{1} \wedge \mathbf{x}_{2} \wedge \mathbf{e}_{\infty 1} \wedge \mathbf{e}_{\infty 2} \wedge \mathbf{e}_{\infty 3} \wedge \mathbf{I}_{\infty b}=\mathbf{x}_{1} \wedge \mathbf{x}_{2} \wedge \mathbf{I}_{\infty}
$$

Looking back, we see that the above expression can also be developed from the CGA point pair (3.7) or understood as another geometric interpretation of the embedding of the CGA line (3.11). Note that the computer algebra construction of all these entities is available using the recently developed software plugin qc3gaTools . hpp ${ }^{3}$.

${ }^{3}$ git clone https://git.renater.fr/garamon.git 
3D quadrics in extended CGA, control points, implicit equations, alignment 15

\section{Dual quadratic surface representation and implicit equations}

The dualization of a primal quadratic surface 9-blade $\mathbf{q}$ after the outer product with $\mathbf{I}_{o}^{\triangleright}$ leads to the dual 1-vector quadratic surface representation $\left(\mathbf{q} \wedge \mathbf{I}_{o}^{\triangleright}\right)^{*}$ of (4.4). Corollary 4.2 can be rephrased as

Proposition 6.1. A point $\mathbf{x}$ lies on the dual quadratic surface $\left(\mathbf{q} \wedge \mathbf{I}_{o}^{\triangleright}\right)^{*}$ iff $\mathbf{x} \cdot(\mathbf{q} \wedge$ $\left.\mathbf{I}_{o}^{\triangleright}\right)^{*}=0$.

This dualization enables us to define axis-aligned quadratic surfaces as vectors in $\mathbb{R}^{9,6}$ by simply using the coefficients of their conventional implicit equations.

\subsection{Some examples of dual quadratic surface representations}

This subsection presents the construction of some specific quadratic surfaces.

6.1.1. Representation of a dual axis-aligned ellipsoid. First, an axis-aligned ellipsoid can be computed as follows:

$$
\left(\mathbf{q} \wedge \mathbf{I}_{o}^{\triangleright}\right)^{*}=\frac{1}{a^{2}} \mathbf{e}_{o 1}+\frac{1}{b^{2}} \mathbf{e}_{o 2}+\frac{1}{c^{2}} \mathbf{e}_{o 3}+\frac{1}{2} \mathbf{e}_{\infty},
$$

where $a, b, c$ are the semi-axis parameters of the ellipsoid. This construction is further illustrated in Figure 6.

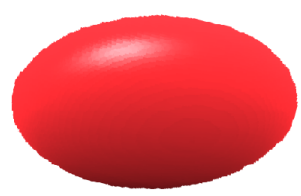

FIGURE 6. Construction of an axis-aligned ellipsoid.

6.1.2. Representation of a dual axis-aligned elliptic cylinder. Another example of a quadratic surface is the cylinder. An elliptic axis-aligned cylinder can easily be defined. A cylinder whose main axis is $(\mathrm{Oz})$ and whose cross section semi-axis are $a$ and $b$ can be defined as follows

$$
\left(\mathbf{q} \wedge \mathbf{I}_{o}^{\triangleright}\right)^{*}=\frac{1}{a^{2}} \mathbf{e}_{o 1}+\frac{1}{b^{2}} \mathbf{e}_{o 2}-\frac{1}{2} \mathbf{e}_{\infty} .
$$

Note that a non-axis aligned elliptic cylinder can be constructed as the outer product of nine points as shown in Figure 7, or it could be obtained from (6.2), applying the geometric transformation versors of [13]. 




FIGURE 7. Construction of a generalized cylinder from nine points.

6.1.3. Representation of a dual axis-aligned hyperbolic paraboloid. Another example of axis-aligned quadratic surface is the hyperbolic paraboloid, also called saddle. It can be defined as:

$$
\left(\mathbf{q} \wedge \mathbf{I}_{o}^{\triangleright}\right)^{*}=\frac{1}{a^{2}} \mathbf{e}_{o 1}-\frac{1}{b^{2}} \mathbf{e}_{o 2}+\frac{1}{2} \mathbf{e}_{3} .
$$

An axis-aligned cone can be dually represented in QCGA as follows:

$$
\left(\mathbf{q} \wedge \mathbf{I}_{o}^{\triangleright}\right)^{*}=\frac{1}{a^{2}} \mathbf{e}_{o 1}+\frac{1}{b^{2}} \mathbf{e}_{o 2}-\mathbf{e}_{o 3} .
$$

6.1.4. Representation of a dual axis-aligned hyperboloid. An axis-aligned hyperboloid of one sheet can be constructed as follows:

$$
\left(\mathbf{q} \wedge \mathbf{I}_{o}^{\triangleright}\right)^{*}=\frac{1}{a^{2}} \mathbf{e}_{o 1}+\frac{1}{a^{2}} \mathbf{e}_{o 2}-\frac{1}{c^{2}} \mathbf{e}_{o 3}-\frac{1}{2} \mathbf{e}_{\infty}
$$

An example of such a quadratic surface is shown in Figure 8. Changing the sign of



FIGURE 8. Construction of a dual hyperboloid of one sheet.

$\frac{1}{2} \mathbf{e}_{\infty}$, the definition of an axis-aligned hyperboloid of two sheets is given by

$$
\left(\mathbf{q} \wedge \mathbf{I}_{o}^{\triangleright}\right)^{*}=\frac{1}{a^{2}} \mathbf{e}_{o 1}+\frac{1}{a^{2}} \mathbf{e}_{o 2}-\frac{1}{c^{2}} \mathbf{e}_{o 3}+\frac{1}{2} \mathbf{e}_{\infty} .
$$

6.1.5. Representation of a dual axis-aligned elliptic paraboloid. An axis-aligned elliptic paraboloid can be defined as

$$
\left(\mathbf{q} \wedge \mathbf{I}_{o}^{\triangleright}\right)^{*}=\frac{1}{a^{2}} \mathbf{e}_{o 1}+\frac{1}{b^{2}} \mathbf{e}_{o 2}+\frac{1}{2} \mathbf{e}_{3} .
$$


6.1.6. Representation of a dual axis-aligned degenerate quadratic surfaces. As previously seen, degenerate quadratic surfaces can also be defined. For example, a pair of planes can be defined as:

$$
\left(\mathbf{q} \wedge \mathbf{I}_{o}^{\triangleright}\right)^{*}=\mathbf{e}_{o 1}-\mathbf{e}_{o 2} .
$$

An illustration of such a pair of planes using QCGA is shown in Figure 9.
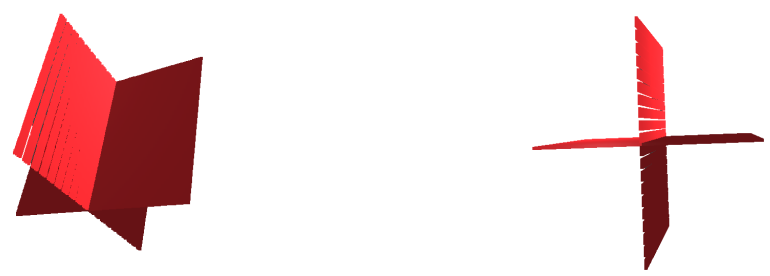

FIGURE 9. Construction of a axis-aligned pair of planes.

Tables 2 and 3 summarize the dual definition of CGA objects, as well as axisaligned quadratic surfaces and degenerate quadratic surfaces.

TABLE 2. Definition of dual CGA objects embedded in QCGA, computed from $S_{\mathrm{C}}$ and $F_{\mathrm{C}}$ of Section 3.2, using $\mathbf{I}_{\mathrm{C}}$ of (2.35). Notation: $\mathbf{c}_{\varepsilon}$ is the Euclidean center position of the sphere, $\mathbf{n}_{\varepsilon}$ the unit normal vector to the plane, $\mathrm{d}$ is the distance of the plane from the origin, $\mathbf{a}_{\varepsilon}$ the direction vector of the line, $\mathbf{m}_{\varepsilon}$ the bivector moment of the line.

\begin{tabular}{|c|l|}
\hline Geometric objects & Dual definition \\
\hline Sphere & $\mathbf{s}_{\mathrm{C}}^{*}=\mathbf{c}_{\varepsilon}-\frac{1}{2} \mathbf{r}^{2} \mathbf{e}_{\infty}$ \\
\hline Plane & $\boldsymbol{\pi}_{\mathrm{C}}^{*}=\mathbf{n}_{\varepsilon}+\mathrm{d}_{\infty}$ \\
\hline Line & $\mathbf{l}_{\mathrm{C}}^{*}=\mathbf{a}_{\varepsilon} \mathbf{I}_{\varepsilon}+\mathbf{e}_{\infty} \mathbf{m}_{\varepsilon} \mathbf{I}_{\varepsilon}$ \\
& $\mathbf{l}_{\mathrm{C}}^{*}=\boldsymbol{\pi}_{\mathrm{C} 1}^{*} \wedge \boldsymbol{\pi}_{\mathrm{C} 2}^{*}$ \\
\hline Circle & $\mathbf{o}_{\mathrm{C}}^{*}=\mathbf{s}_{\mathrm{C} 1}^{*} \wedge \mathbf{s}_{\mathrm{C} 1}^{*}$ \\
& $\mathbf{o}_{\mathrm{C}}^{*}=\mathbf{s}_{\mathrm{C}}^{*} \wedge \boldsymbol{\pi}_{\mathrm{C}}$ \\
\hline Point pair & $\mathbf{p}_{p \mathrm{C}}^{*}=\mathbf{s}_{\mathrm{C}}^{*} \wedge \mathbf{l}_{\mathrm{C}}^{*}$ \\
& $\mathbf{p}_{p \mathrm{C}}^{*}=\mathbf{o}_{\mathrm{C} 1}^{*} \wedge \mathbf{o}_{\mathrm{C} 2}^{*}$ \\
& $\mathbf{p}_{p \mathrm{C}}^{*}=\mathbf{s}_{\mathrm{C} 1}^{*} \wedge \mathbf{s}_{\mathrm{C} 2}^{*} \wedge \mathbf{s}_{\mathrm{C} 3}^{*}$ \\
\hline
\end{tabular}


TABLE 3. Definition of dual axis-aligned quadratic surfaces using QCGA.

\begin{tabular}{|c|l|}
\hline Geometric object & Dual vector definition \\
\hline Ellipsoid & $\left(\mathbf{q} \wedge \mathbf{I}_{o}^{\triangleright}\right)^{*}=\frac{1}{a^{2}} \mathbf{e}_{o 1}+\frac{1}{b^{2}} \mathbf{e}_{o 2}+\frac{1}{c^{2}} \mathbf{e}_{o 3}+\frac{1}{2} \mathbf{e}_{\infty}$ \\
\hline Cone & $\left(\mathbf{q} \wedge \mathbf{I}_{o}^{\triangleright}\right)^{*}=\frac{1}{a^{2}} \mathbf{e}_{o 1}+\frac{1}{b^{2}} \mathbf{e}_{o 2}-\mathbf{e}_{o 3}$ \\
\hline Cylinder & $\left(\mathbf{q} \wedge \mathbf{I}_{o}^{\triangleright}\right)^{*}=\frac{1}{a^{2}} \mathbf{e}_{o 1}+\frac{1}{b^{2}} \mathbf{e}_{o 2}-\frac{1}{2} \mathbf{e}_{\infty}$ \\
\hline Hyperbolic paraboloid & $\left(\mathbf{q} \wedge \mathbf{I}_{o}^{\triangleright}\right)^{*}=\frac{1}{a^{2}} \mathbf{e}_{o 1}-\frac{1}{b^{2}} \mathbf{e}_{o 2}+\frac{1}{2} \mathbf{e}_{3}$ \\
\hline Elliptic paraboloid & $\left(\mathbf{q} \wedge \mathbf{I}_{o}^{\triangleright}\right)^{*}=\frac{1}{a^{2}} \mathbf{e}_{o 1}+\frac{1}{b^{2}} \mathbf{e}_{o 2}+\frac{1}{2} \mathbf{e}_{3}$ \\
\hline $\begin{array}{c}\text { Hyperboloid } \\
\text { one sheet }\end{array}$ & $\left(\mathbf{q} \wedge \mathbf{I}_{o}^{\triangleright}\right)^{*}=-\frac{1}{a^{2}} \mathbf{e}_{o 1}-\frac{1}{a^{2}} \mathbf{e}_{o 2}+\frac{1}{c^{2}} \mathbf{e}_{o 3}+\frac{1}{2} \mathbf{e}_{\infty}$ \\
two sheets & $\left(\mathbf{q} \wedge \mathbf{I}_{o}^{\triangleright}\right)^{*}=-\frac{1}{a^{2}} \mathbf{e}_{o 1}-\frac{1}{a^{2}} \mathbf{e}_{o 2}+\frac{1}{c^{2}} \mathbf{e}_{o 3}-\frac{1}{2} \mathbf{e}_{\infty}$ \\
\hline Pair of planes & $\mathbf{q}^{*}=\mathbf{e}_{o 1}-\mathbf{e}_{o 2}$ \\
\hline
\end{tabular}

Table 4 details a class of objects that can be handled using QCGA. Table 5 summarizes some definitions of axis-aligned primal objects constructed from points in QCGA.

TABLE 4. Definition of primal geometric objects using QCGA.

\begin{tabular}{|l|c|}
\hline Round object $($ sphere, circle,..$)$ & $\mathbf{q}=\mathbf{x}_{1} \wedge \mathbf{x}_{2} \wedge \cdots \wedge \mathbf{I}_{\infty}^{\triangleright}$ \\
\hline Flat object (plane, line, $\ldots)$ & $\mathbf{q}=\mathbf{x}_{1} \wedge \mathbf{x}_{2} \wedge \cdots \wedge \mathbf{e}_{\infty} \wedge \mathbf{I}_{\infty}^{\triangleright}$ \\
\hline Axis-aligned quadratic surface & $\mathbf{q}=\mathbf{x}_{1} \wedge \cdots \wedge \mathbf{x}_{6} \wedge \mathbf{I}_{\infty b}$ \\
\hline General quadratic surface & $\mathbf{q}=\mathbf{x}_{1} \wedge \mathbf{x}_{2} \wedge \mathbf{x}_{2} \cdots \wedge \mathbf{x}_{9}$ \\
\hline
\end{tabular}

The construction of axis aligned and origin centred quadratic surfaces based on their implicit equation coefficients is seen to be very straightforward. The availability of versors for rotation, translation and scaling [13] allows then to begin with aligned quadratic surfaces centred at the origin and to subsequently move them to arbitrary position, freely change their orientation by rotation, and moreover scale them arbitrarily.

\section{Intersections}

One of the most fascinating properties of QCGA, is that like in CGA, all objects can be intersected by simply taking the outer products of their duals. That is, any number of linearly independent round or flat embedded CGA objects in QCGA 
TABLE 5. Definition of some primal axis-aligned quadratic surfaces using QCGA.

\begin{tabular}{|l|c|}
\hline $\begin{array}{l}\text { Ellipsoids } \\
\text { Paraboloids }\end{array}$ & $\mathbf{q}=\mathbf{x}_{1} \wedge \cdots \wedge \mathbf{x}_{6} \wedge \mathbf{I}_{\infty b}$ \\
Hyperbolic paraboloids & $\mathbf{q}=\mathbf{x}_{1} \wedge \cdots \wedge \mathbf{x}_{5} \wedge\left(\mathbf{e}_{\infty 1}-\mathbf{e}_{\infty 2}\right) \wedge \mathbf{I}_{\infty b}$ \\
\hline Spheroids & $\mathbf{q}=\mathbf{x}_{1} \wedge \mathbf{x}_{2} \wedge \mathbf{x}_{3} \wedge \mathbf{x}_{4} \wedge \mathbf{x}_{5} \wedge \mathbf{e}_{\infty 3} \wedge \mathbf{I}_{\infty b}$ \\
\hline $\begin{array}{l}\text { Cylinders } \\
\text { Elliptic cylinders }\end{array}$ & \\
\hline
\end{tabular}

and any number of quadratic surfaces after wedging with $\mathbf{I}_{o}^{\triangleright}$, can be intersected by computing the dual of the outer product of duals as follows (see [13])

$$
\left(\text { intersect } \wedge \mathbf{I}_{o}^{\triangleright}\right)^{*}=\left(\mathbf{A} \wedge \mathbf{I}_{o}^{\triangleright}\right)^{*} \wedge\left(\mathbf{B} \wedge \mathbf{I}_{o}^{\triangleright}\right)^{*} \wedge \ldots \wedge\left(\mathbf{Z} \wedge \mathbf{I}_{o}^{\triangleright}\right)^{*} .
$$

The criterion for a general point $\mathbf{x}$ to be on the intersection is

$$
\mathbf{x} \cdot\left(\text { intersect } \wedge \mathbf{I}_{o}^{\triangleright}\right)^{*}=0, \quad \text { intersect }=-\frac{1}{3}\left(\left(\text { intersect } \wedge \mathbf{I}_{o}^{\triangleright}\right)^{*} \mathbf{I}\right)\left\lfloor\mathbf{I}_{\infty}^{\triangleright} .\right.
$$

For cases in which one object is completely included in another object (like a line in a plane), the proper meet operation has to be defined by taking into account the subspace spanned by the join of the two objects [15].

\section{Conclusion}

This paper presented a development of QCGA to represent and manipulate quadratic surfaces in extended geometric algebras, in particular, aligned or symmetric quadratic surfaces. After recalling the main ideas of QCGA [2], together with the null basis vector modifications of [13], we presented a detailed set of algebraic constructions and notations. This then allowed us to represent both embedded objects of CGA and quadratic surfaces of QCGA in a constructive and intuitive way. Furthermore, quadratic surfaces are now represented more concisely and efficiently from either their implicit forms, implicit axis aligned and origin centred forms followed by geometric versor transformations, or their control points. The intersection of all these objects can easily be computed. In the future, we plan to extend this approach to represent quadratic surfaces also to cubic surfaces. Finally, note that the examples presented in this paper were computed and visualized efficiently using the new $\mathrm{C}++$ library called Garamon in [1].

\section{Acknowledgments}

We do thank the organizers of the international conference AGACSE 2018 for the inspiring event held in the summer of 2018 in Campinas, Brazil, that greatly facilitated our collaboration. EH wants to thank God Soli Deo Gloria, and invite all 
readers of this work to take the Creative Peace License ${ }^{4}$ into consideration, when applying this researchess.

\section{References}

[1] Breuils, S., Nozick, V., AND Fuchs, L. Garamon: Geometric algebra library generator. submitted to Advances in Applied Clifford Algebras.

[2] Breuils, S., Nozick, V., Sugimoto, A., AND Hitzer, E. Quadric conformal geometric algebra of $\mathbb{R}^{9,6}$. Advances in Applied Clifford Algebras 28, 35 (March 2018), 16 pages.

[3] Buchiolz, S., Tachibana, K., And Hitzer, E. Optimal learning rates for Clifford neurons. In International conference on artificial neural networks (2007), Springer, pp. 864-873.

[4] Dorst, L., And VAn Den BoomgaArd, R. An analytical theory of mathematical morphology, 1993. Available at https://www.researchgate.net/profile/Leo_ Dorst/publication/2811399_An_Analytical_Theory_of_Mathematical_ Morphology/links/09e41510c0f213ff3b000000.pdf.

[5] Du, J., Goldman, R., And Mann, S. Modeling 3D Geometry in the Clifford Algebra $\mathbb{R}^{4,4}$. Advances in Applied Clifford Algebras 27, 4 (Dec 2017), 3039-3062.

[6] Easter, R.B. And Hitzer, E. Double conformal geometric algebra. Advances in Applied Clifford Algebras 27, 3 (April 2017), 2175-2199.

[7] Goldman, R., And MAnn, S. R(4, 4) as a computational framework for 3dimensional computer graphics. Advances in Applied Clifford Algebras 25, 1 (March 2015), 113-149.

[8] Gregory, A. L., Lasenby, J., And Agarwal, A. The elastic theory of shells using geometric algebra. Royal Society open science 4, 3 (2017), 170065.

[9] HestenEs, D. The zitterbewegung interpretation of quantum mechanics. Foundations of Physics 20, 10 (October 1990), 1213-1232.

[10] Hestenes, D. New foundations for classical mechanics, vol. 15. Springer Science \& Business Media, 2012.

[11] Hestenes, D., AND SobCzyK, G. Clifford algebra to geometric calculus: a unified language for mathematics and physics, vol. 5. Springer Science \& Business Media, 2012.

[12] HitZer, E. Geometric operations implemented by conformal geometric algebra neural nodes. Preprint arXiv:1306.1358 (2013).

[13] Hitzer, E. Three-dimensional quadrics in conformal geometric algebras and their versor transformations. Advances in Applied Clifford Algebras 29, 46 (July 2019), 16 pages. doi: 10.1007/s00006-019-0964-1, preprint: http://vixra.org/abs/ 1902.0401.

[14] Hitzer, E., Tachibana, K., Buchholz, S., and Yu, I. Carrier method for the general evaluation and control of pose, molecular conformation, tracking, and the like. Advances in Applied Clifford Algebras 19, 2 (July 2009), 339-364.

[15] LI, H. Invariant algebras and geometric reasoning. World Scientific, 2008.

${ }^{4}$ E. Hitzer, Creative Peace License, https://gaupdate.wordpress.com/2011/12/14/ the-creative-peace-license-14-dec-2011/. 
3D quadrics in extended CGA, control points, implicit equations, alignment 21

[16] LuO, W., Hu, Y., Yu, Z., YuAN, L., AND LÜ, G. A Hierarchical Representation and Computation Scheme of Arbitrary-dimensional Geometrical Primitives Based on CGA. Advances in Applied Clifford Algebras 27, 3 (September 2017), 1977-1995.

[17] Papaefthymiou, M., and Papagiannakis, G. Real-time rendering under distant illumination with conformal geometric algebra. Mathematical Methods in the Applied Sciences 41, 11 (July 2018), 4131-4147.

[18] Sangwine, S. J., AND Hitzer, E. Clifford multivector toolbox (for MATLAB). Advances in Applied Clifford Algebras 27, 1 (2017), 539-558.

[19] VINCE, J. Geometric algebra for computer graphics. Springer Science \& Business Media, 2008.

[20] Zhu, S., Yuan, S., Li, D., Luo, W., Yuan, L., AND Yu, Z. Mvtree for hierarchical network representation based on geometric algebra subspace. Advances in Applied Clifford Algebras 28, 39 (April 2018), 15 pages.

Stéphane Breuils

Laboratoire d'Informatique Gaspard-Monge, Equipe A3SI,

UMR 8049, Université Paris-Est Marne-la-Vallée, France

e-mail: stephane.breuils@u-pem.fr

Laurent Fuchs

Laboratoire XLIM-ASALI, UMR CNRS 7252, Université de Poitiers, France

e-mail: Laurent.Fuchs@univ-poitiers.fr

Eckhard Hitzer

International Christian University, Tokyo 181-8585, Japan

e-mail: hitzer@icu.ac.jp

Vincent Nozick

Laboratoire d'Informatique Gaspard-Monge, Equipe A3SI,

UMR 8049, Université Paris-Est Marne-la-Vallée, France

e-mail: vincent.nozickeu-pem.fr

Akihiro Sugimoto

National Institute of Informatics, Tokyo 101-8430, Japan

e-mail: sugimoto@nii.ac.jp 